\title{
Surgical Versus Non-surgical Management of Lumbar Spinal Stenosis
}

\author{
Paul E. Kaloostian · Ziya L. Gokaslan
}

Published online: 16 May 2013

(c) Springer Science+Business Media New York 2013

\begin{abstract}
Lumbar spinal stenosis is a common degenerative disorder affecting the lumbar spine. It is most commonly associated with facet arthropathy and hypertrophied ligamentum flavum, with a small subset due to large acute disc herniations. Patients may present with a wide variety of neurological signs and symptoms, ranging from asymptomatic presentation to neurogenic claudication, and in the most severe form, cauda equina syndrome. Methods of diagnosis include careful history and physical examination, along with magnetic resonance imaging. Non-surgical management includes physical therapy, pain management, and epidural steroid injections. Surgical treatment includes lumbar laminectomy with or without fusion. A review of the causes and treatment options for lumbar stenosis is described in this manuscript.
\end{abstract}

Keywords Spinal stenosis - Lumbar stenosis . Laminectomy · Interspinous spacers - Claudication . Lumbar decompression

\section{Introduction}

Lumbar spinal stenosis (LSS) is one of the most common pathological conditions affecting the lumbar spine [1-3]. It is most commonly seen in patients above the age of 60 , though this condition may affect patients at any age above 30. The incidence of LSS has been increasing over the past

P. E. Kaloostian $(\bowtie) \cdot$ Z. L. Gokaslan

The Johns Hopkins Hospital, 600 North Wolfe Street, Baltimore, MD 21287, USA

e-mail: paulkaloostian@hotmail.com

Z. L. Gokaslan

e-mail: zgokasl1@jhmi.edu few decades, with an estimated 64 million people above the age of 65 being diagnosed by the year 2025. Besides the dramatic disability imposed upon patients, the economic impact to the healthcare system worldwide is significant and increasing [1-5].

\section{Pathophysiology}

LSS is classically defined as a narrowing of the central vertebral canal adjacent to one or more lumbar vertebrae. The most common causes of LSS include degenerative disease of the spine and spondylosis. Other less common causes of LSS include stenosis from tumor, hemorrhage, large synovial cysts, and epidural lipomatosis [6-8]. Degenerative disease of the lumbar spine is a progressive phenomenon that advances with increasing age; hence the increased incidence in the elderly. The lumbar spine has a significant responsibility of accepting the stress placed upon it by the entire weight of the body. This immense load sharing capacity upon the lumbar spine, and particularly the lower lumbar spine, advances the degenerative process at the level of the facet joints. Activities such as running and jumping over long periods of time place added stress upon the lumbar spine and accelerate the degenerative process that occurs normally with advancing age $[4,9,10]$. Other iatrogenic causes such as traumatic injury to the spine as well as smoking increase the degenerative capacity of the spine.

Over time, with repeated stress upon the lumbar spine, the facet joints and ligamentum flavum begin to change morphology to adapt to the continued forces upon the lumbar spine. The facet joints begin to hypertrophy bilaterally from continued motion at the joints. The ligamentum flavum also hypertrophies within the spinal canal. Both of 


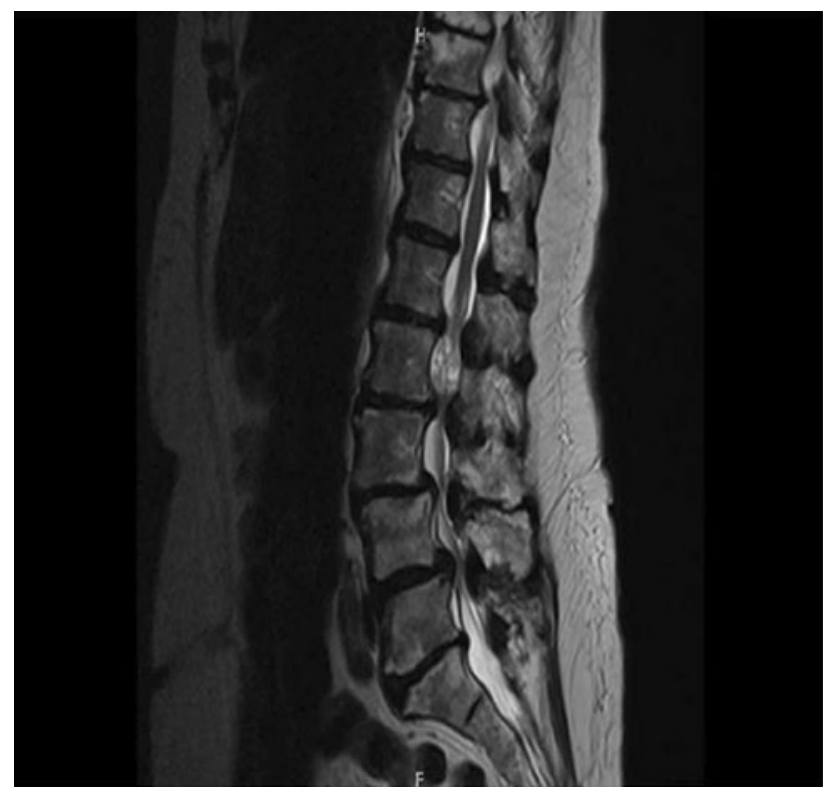

Fig. 1 Sagittal MRI lumbar spine in a 45-year-old male with neurogenic claudication and severe low back pain demonstrating severe lumbar spinal stenosis at every level due to facet arthropathy, ligamentum hypertrophy, and degenerative disc disease. He required a multilevel laminectomy with medial facetectomy and a posterolateral fusion

these adaptations decrease the amount of space within the confined spinal canal, bordered at all sides by bony structures, with the lamina posteriorly, pedicles laterally, and vertebral body anteriorly. Similar to the Monro-Kellie doctrine in cranial mass effect, there is a limited space within the spinal canal, and any added mass within the canal will cause a shift of its contents. This shift will reach a point where additional mass effect will cause symptomatic compression of existing neural and vascular structures within the canal, leading to neurological signs and symptoms. The degenerative hypertrophic changes within the lumbar spinal canal from facet arthropathy and ligamentum hypertrophy will reach a critical point where the existing neural and vascular structures are compromised, leading to a variety of symptoms (Fig. 1). It is typically at this point that the patient will begin to seek medical attention $[4-6,9$, $10]$.

\section{Neurological Presentation}

Neurological symptoms from lumbar stenosis vary, depending on the degree of spinal canal compromise. For example, some patients with early mild lumbar stenosis will be asymptomatic or present with only minimal back pain. Others with slightly more advanced lumbar stenosis may present with back pain and occasional radicular leg pain $[11-13,14 \bullet \bullet, 15]$. Finally, those with very advanced lumbar stenosis will typically present with back pain and neurogenic claudication. These patients will present with an inability to walk long distances, typically 1-2 blocks, due pain or discomfort in their legs. They will describe their legs feeling very heavy, at which point they must sit down for a certain period of time. After sitting down, the spinal canal opens up sufficiently enough to allow the compressed neural and vascular structures to recover. The patient can then stand back up and ambulate for a few more blocks until the symptoms arise again. Patients often describe that while they are grocery shopping, they often have the need to lean forward on the shopping cart, which opens up the spinal canal and allows them to ambulate for longer distances without their legs feeling heavy. Those patients with the most advanced lumbar stenosis will present with cauda equina syndrome, with the classic findings of perineal or saddle anesthesia, bowel/bladder incontinence, and/or lower extremity weakness. This syndrome is often due to a large disc herniation compressing the cauda equina significantly and acutely. This is a neurosurgical emergency and urgent surgical intervention within $24 \mathrm{~h}$ after symptom onset is recommended to prevent permanent neural injury [5-13, 14••, 15].

\section{Diagnostic Modalities}

Diagnosis of lumbar stenosis is based upon a careful history, physical examination, and diagnostic imaging modalities. Obtaining a careful history from the patient will elucidate an inability to walk long distances without the legs giving out on the patient. Also, the patient will often describe that sitting down or leaning forward improves their symptoms [12, 13, 14••, 15-19]. Physical examination is often quite normal, with intact motor and sensory function, and symmetric reflexes. Bowel and bladder function is typically intact. The only exception is severe lumbar stenosis with cauda equina syndrome, where patients present with acute onset of lower extremity weakness, bowel and bladder dysfunction, and perineal numbness [15-19]. The gold standard diagnostic modality is magnetic resonance imaging (MRI) which demonstrates spinal stenosis due to ligamentum hypertrophy and facet arthropathy. Computerized tomography (CT) is helpful to inspect bony osteophytes along the lumbar spinal canal contributing to stenosis. Flexion-extension X-rays of the lumbar spine are useful to identify any spondylolisthesis, or abnormal excessive motion of the lumbar spine due to facet pathology, which may increase lumbar stenosis upon movement. X-rays may also detect scoliosis of the lumbar spine, which can change surgical management. Electromyography and nerve conduction studies can detect radiculopathy, and whether nerve injury is acute or chronic. 
Finally, it is important to rule out vascular claudication from atherosclerotic vascular disease as it can mimic symptomatic neurogenic claudication. Patients with vascular claudication will have similar symptoms, but on examination may have a reduced or absent pulse in the affected lower extremity and skin stigmata showing peripheral vascular disease, such as venous engorgement, pitting edema, and very slow capillary refill. An arterial and venous ultrasound and angiogram are recommended to rule out vascular claudication [18-23].

\section{Non-surgical Management}

A variety of treatment options exist for LSS. Trials of conservative management are typically recommended after initial diagnosis with hopes of diminishing pain, increasing lower extremity strength, and improving quality of life. Physical therapy sessions are recommended initially to increase range of motion and strength in lower extremities, as well as to increase core muscle strength. Spinal orthotics can provide symptomatic relief of neurogenic claudication (especially in people with achondroplasia) by reducing lumbar lordosis. This can increase the volume of the lumbar spinal canal, thereby reducing symptoms of neurovascular impingement [24].

Management of low back pain is typically with antiinflammatory medications as well as low dose opioid therapy. Pain management specialists or physiatrists should be consulted to aid in this process. Epidural steroid injections either translaminar or transforaminal should be attempted as part of conservative management. The mechanism of action is though to be related to the steroids diminishing the inflammatory response in the epidural space due to ligament and facet hypertrophy, and thus increasing space within the spinal canal. However, patients often require repeated injections due to the temporary nature of steroids within the epidural space. Some patients may not benefit at all from the injections, while others benefit quite dramatically, with an increased length of steroid pain relief and increased spacing of steroid injections [23, 25, 26]. Most physicians that perform the injections will not perform more than 3-4 injections within a year. While some studies have demonstrated benefit of epidural steroid injections, other studies have shown contrary results. For example, some studies have shown that long term epidural steroid injections within the lumbar spine has increased complication rates for those who undergo surgery thereafter. The authors describe a more complicated lumbar decompression due to adhesions, which may lead to cerebrospinal fluid leak as a complication. Also, the authors noted an increased operative time and increased blood loss in the group treated with epidural steroids. The reasons for this remain unclear. The authors hypothesize that the epidural steroids cause increased adhesions and scarring within the epidural space. Also, they describe increased epidural lipomatosis as a complication of long-term steroid use. Finally, the role of steroids as causing direct injury to neural structures remains a possibility, though no study has proven this [27-32].

\section{Surgical Management}

If the patient fails the above conservative strategies, there are a variety of surgical options available, most of which have proven to be quite beneficial. The classic surgical treatment, and one that has withstood the test of time, is the typical lumbar decompression. This surgical modality involves placing the patient in a prone position on gel pads and with the use of a high-speed drill and Kerrison rongeurs involves performing bilateral laminectomies with medial facetectomies and foraminotomies. Removing only the medial aspect of the large hypertrophied facets avoids destabilizing the facet joints. Patients with symptoms from epidural tumor, synovial cyst, lipomatosis, or hemorrhage require additional steps of removing the offending pathology. The number of levels operated on will depend on what the MRI findings are in each particular patient. Surgery has been shown to be beneficial if symptoms have been going on after 3-6 months of conservative treatment [33••, 34••]. This may also be performed in a minimally invasive fashion through a mini-open incision. Drawbacks of the minimally invasive approach involve inadequate decompression and risk of cerebrospinal fluid leak with difficulty closing the dural tear intra-operatively. Benefits of the minimally invasive approach include shorter hospital stay, decreased pain post-operatively, and smaller incision size [35]. If the patient has a severe degree of mechanical back pain worsened with flexion and extension, with flexion-extension X-rays demonstrating spondylolisthesis greater than $3 \mathrm{~mm}$, then lumbar decompression is augmented with spinal instrumentation and posterolateral fusion. The instrumentation procedure involves pedicle screw insertion at the appropriate levels based on MRI and CT findings, as well as posterolateral fusion and arthrodesis at the facet joints with autograft and allograft material. During the decompression, the neural foramina are opened up with Kerrison rongeurs to remove excess bony or ligament compression. Large disc herniations anteriorly compressing the thecal sac and contributing to lumbar stenosis can be removed by opening up the disc space and pulling out disc fragments. Anterior fusion may also be performed by placing interbody devices into the disc space to allow for circumferential fusion in addition to the posterior instrumentation. 
Patients with scoliosis add another degree of difficulty to surgical treatment. Surgical treatment will depend on patient symptoms and degree of scoliosis. Often times, focal lumbar decompression without scoliosis treatment will be sufficient, particularly for the elderly patient population.

\section{Newer Surgical Management Strategies}

Other more recent methods of surgical treatment for patients with lumbar stenosis include the X-Stop implants, Superion Interspinous Spacer, and PDS Percudyn System implants [36-41]. These devices have proven useful for patients who are at high risk for surgery, such as the elderly population, as the operative time is significantly shorter with less blood loss. X Stop implants and Superion Interspinous Spacers are a novel method of increasing the interlaminar distance by implanting a device between the spinous processes. This will allow for an increase in the spinal canal diameter and improve symptoms of neurogenic claudication. While some studies have demonstrated benefit of this implant, other studies have found no improvement with the use of the X Stop device. The PDS Percudyn System is a newer, minimally invasive approach that involves bilateral transpedicular facet augmentation with dynamic stabilization devices aimed at preserving motion at the joints while increasing spinal canal diameter. These are posterior stabilizing implants that do not involve fusion, with the benefit of absorbing and modifying the load transfer through anatomical structures. This will avoid complications associated with fusion, such as decreased bone mineral density, pseudoarthrosis, and other stressshielding effects.

\section{Surgical Complications}

Surgical treatments are not without a risk of complications. For example, standard lumbar decompression may cause cerebrospinal fluid leak that may require re-operation if the dural tear is not sewn appropriately. Also, there is a risk of neural injury to the nerve roots from decompression causing post-operative radiculopathy with weakness or numbness. Patients may develop wound infections and can have significant intra-operative blood loss from epidural venous bleeding requiring transfusion with all of the potential transfusion-associated complications. Finally, patients who undergo standard decompression may be left with spinal instability due to pre-existing spondylolisthesis or iatrogenic removal of too much of the facet joint and pars interarticularis intra-operatively, requiring a second surgery for instrumentation and fusion [14••, 15-19].

\section{Conclusion}

In summary, LSS is a common degenerative disorder affecting the lumbar spine. A combination of facet arthropathy and ligamentum flavum hypertrophy most commonly contribute to this condition. Patients may present asymptomatically, with neurogenic claudication, or cauda equina syndrome. Non surgical and surgical treatments vary, each with pros and cons. Newer novel methods of surgical intervention are promising for long term and less risky treatment of patients with LSS.

Disclosure PE Kaloostian and ZL Gokaslan declare no conflicts of interest.

\section{References}

Papers of particular interest, published recently, have been highlighted as:

•- Of major importance

1. Ciol MA, Deyo RA, Howell E, et al. An assessment of surgery for spinal stenosis: time trends, geographic variations, complications, and reoperations. J Am Geriatr Soc. 1996;44:285-90.

2. Katz JN, Harris MB. Clinical practice: lumbar spinal stenosis. N Engl J Med. 2008;358:818-25.

3. Atlas SJ, Delitto A. Spinal stenosis: surgical versus nonsurgical treatment. Clin Orthop Relat Res. 2006;443:198-207.

4. Porter RW. Spinal stenosis and neurogenic claudication. Spine. 1996;21:2046-52.

5. Simotas AC, Dorey FJ, Hansraj KK, et al. Nonoperative treatment for lumbar spinal stenosis: clinical outcome results and a 3-year survivorship analysis. Spine. 2000;25:197-203 (discussions 203-204).

6. Sekiguchi M, Kikuchi S. Experimental studies of lumbar spinal stenosis. Clin Calcium. 2005;15:51-6.

7. Benoist $M$. The natural history of lumbar degenerative spinal stenosis. Jt Bone Spine. 2002;69:450-7.

8. Weinstein JN, Tosteson TD, Lurie JD, et al. Surgical versus nonsurgical therapy for lumbar spinal stenosis. N Engl J Med. 2008;358:794-810.

9. Deyo RA. Treatment of lumbar spinal stenosis: a balancing act. Spine J. 2010;10:625-7.

10. Atlas SJ, Keller RB, Robson D, et al. Surgical and nonsurgical management of lumbar spinal stenosis: four-year outcomes from the marine lumbar spine study. Spine. 2000;25:556-62.

11. Zak PJ. Surgical management of spinal stenosis. Phys Med Rehabil Clin N Am. 2003;14(1):143-4.

12. Morishita $Y$, Hida $S$, Naito $M$, et al. Neurogenic intermittent claudication in lumbar spinal canal stenosis: the clinical relationship between the local pressure of the intervertebral foramen and the clinical findings in lumbar spinal canal stenosis. J Spinal Disord Tech. 2009;22(2):130-4.

13. Kleinstuck FS, Grob D, Lattig F, et al. The influence of preoperative back pain on the outcome of lumbar decompression surgery. Spine. 2009;34(11):1198-203.

14. • Genevay S, Atlas SJ. Lumbar spinal stenosis. Best Pract Res Clin Rheumatol. 2010;24(2):253-65. This study critically analyzes the diagnostic and treatment options for symptomatic lumbar stenosis. 
15. Okoro T, Qureshi A, Sell B, et al. The accuracy of assessment of walking distance in the elective spinal outpatients setting. Eur Spine J. 2010;19(2):279-82.

16. Arbit E, Pannullo S. Lumbar stenosis: a clinical review. Clin Orthop Relat Res. 2001;384:137-43.

17. Hart LG, Deyo RA, Cherkin DC. Physician office visits for low back pain: frequency, clinical evaluation, and treatment patterns from a U.S. national survey. Spine. 1995;20(1):11-9.

18. Long DM, BenDebba M, Torgerson WS, et al. Persistent back pain and sciatica in the United States: patient characteristics. J Spinal Disord. 1996;9(1):40-58.

19. Johnson KE. Lumbar spinal stenosis: a retrospective study of 163 cases in southern Sweden. Acta Orthop Scand. 1995;66(5):403-5.

20. Sheehan NJ. Magnetic resonance imaging for low back pain: indications and limitations. Postgrad Med J. 2010;86(1016): $374-8$.

21. Cousins JP, Haughton VM. Magnetic resonance imaging of the spine. J Am Acad Orthop Surg. 2009;17(1):22-30.

22. Tracy JA, Bartleson JD. Cervical spondylotic myelopathy. Neurologist. 2010;16(3):176-87.

23. Kiers L, Desmond P. Magnetic resonance imaging in subacute combined degeneration of the spinal cord. J Clin Neurosci. 1999;6(1):49-50.

24. Siebens AA, Hungerford DS, Kirby NA. Achondroplasia: effectiveness of an orthosis in reducing deformity of the spine. Arch Phys Med Rehabil. 1987;68(6):384-8.

25. de Tran QH, Duong S, Finalyson RJ. Lumbar spinal stenosis: a brief review of the nonsurgical management. Can J Anaesth. 2010;57(7):694-703.

26. Djurasovic M, Glassman SD, Carreon LY, et al. Contemporary management of symptomatic lumbar spinal stenosis. Orthop Clin N Am. 2010;41(2):183-91.

27. Briggs VG, Li W, Kaplan MS, et al. Injection treatment and back pain associated with degenerative lumbar spinal stenosis in older adults. Pain Phys. 2010;13:E347-55.

28. Lee JW, Myung JS, Park KW, et al. Fluoroscopically-guided caudal epidural injection for management of degenerative lumbar spinal stenosis: short-term and long-term results. Skelet Radiol. 2010;39:691-9.

29. Koc Z, Ozcakir S, Sivrioglu K, et al. Effectiveness of physical therapy and epidural steroid injections in lumbar spinal stenosis. Spine. 2009;34:985-9.

30. Cuckler JM, Bernini PA, Wiesel SW, et al. The use of epidural steroids in the treatment of lumbar radicular pain: a prospective, randomized, double-blind study. J Bone Jt Surg Am. 1985;67:63-6.
31. Fukusaki M, Kobayashi I, Hara T, et al. Symptoms of spinal stenosis do not improve after epidural steroid injection. Clin J Pain. 1998;14:148-51.

32. Danielson KD, Harrast MA. Focal spinal epidural lipomatosis after a single epidural steroid injection. Phys Med Rehabil. 2011;3:590-3.

33. - Burnett MG, Stein SC, Bartels RH. Cost-effectiveness of current treatment strategies for lumbar spinal stenosis: nonsurgical care, laminectomy, and X-STOP. J Neurosurg Spine. 2010;13(1):39-46. This article describes the current methods of treatment including conservative management, surgical treatment and minimally invasive techniques.

34. • Kovacs FM, Urrutia G, Alarcon JD. Surgery versus conservative treatment for symptomatic lumbar spinal stenosis: a systematic review of randomized controlled trials. Spine. 2011;36(20):E1335-51. This study is a review of randomized trials comparing surgical versus non surgical management strategies demonstrating the pros and cons of each method of treatment.

35. Gerszten PC, Welch WC. Spine: minimally invasive techniques. Prog Neurol Surg. 2006;19:135-51.

36. Shabat S, Miller LE, Block JE, et al. Minimally invasive treatment of lumbar spinal stenosis with a novel interspinous spacer. Clin Interv Aging. 2011;6:227-33.

37. Masala S, Tarantino U, Nano G, et al. Lumbar spinal stenosis minimally invasive treatment with bilateral transpedicular facet augmentation system. Cardiovasc Interv Radiol. 2012; Epub ahead of print. doi:10.1007/s00270-012-0478.

38. Zucherman JF, Hsu KY, Hartjen CA, et al. A prospective randomized multicentre study for the treatment of lumbar spinal stenosis with the X-STOP interspinous implant: 1 year results. Eur Spine J. 2004;13:22-31.

39. Verhoof OJ, Bron JL, Wapstra FH, et al. High failure rate of the interspinous distraction device (X-STOP) for the treatment of lumbar spinal stenosis caused by degenerative spondylolisthesis. Eur Spine J. 2008;17:188-92.

40. Brussee P, Hauth J, Donk RD, et al. Self-read evaluation of outcome of the implantation of interspinous process distraction (X-STOP) for neurogenic claudication. Eur Spine J. 2008;17(2): 200-3.

41. Barbagallo GM, Olindo G, Corino KL, et al. Analysis of complication in patients treated with the X-STOP interspinous process decompression system: proposal for a novel anatomic scoring system for patient selection and review of the literature. Neurosurgery. 2009;65(1):111-2. 